\title{
Stress perfusion cardiovascular magnetic resonance and serial fractional flow reserve assessment of the left anterior descending artery in patients undergoing right coronary artery chronic total occlusion revascularization
}

\author{
Richard E. Jones ${ }^{1}$, Grigoris V. Karamasis ${ }^{1,2}$, Jason N. Dungu ${ }^{1}$, Shah R. Mohdnazri ${ }^{1,2}$,
} Firas Al-Janabi ${ }^{1,2}$, Daniel J. Hammersley ${ }^{3}$, Sanjay K. Prasad ${ }^{3}$, Kare H. Tang ${ }^{1}$, Paul A. Kelly ${ }^{1}$, Swamy Gedela ${ }^{1}$, John R. Davies ${ }^{1,2}$, Thomas R. Keeble ${ }^{1,2}$

${ }^{1}$ The Essex Cardiothoracic Centre, Basildon and Thurrock University

Hospitals NHS Foundation Trust, Basildon, United Kingdom ${ }^{2}$ School of Medicine, Anglia Ruskin University, Bishop Hall Lane, Chelmsford, United Kingdom

${ }^{3}$ National Heart and Lung Institute, Imperial College London, United Kingdom

\begin{abstract}
Background: Fractional flow reserve (FFR) assessment of remote arteries, in the context of a bystander chronic total occlusion (CTO), can lead to false positive results. Adenosine stress cardiovascular magnetic resonance (CMR) evaluates perfusion defects across the entire myocardium and may therefore be a reliable tool in the work-up of remote lesions in CTO patients. The IMPACT-CTO study investigated donor artery invasive physiology before, immediately post, and at 4 months following right coronary artery (RCA) CTO percutaneous coronary intervention (PCI). The aim of this subanalysis was to assess the concordance between baseline perfusion CMR and serial FFR evaluation of left anterior descending artery (LAD) ischemia in patients from the IMPACT-CTO study.

Methods: Baseline adenosine stress CMR examinations from 26 patients were analyzed for qualitative evidence of LAD ischemia. The results were correlated with the serial LAD FFR measurements.

Results: The present findings demonstrated that before RCA CTO PCI, there was $62 \%$ agreement between perfusion CMR and FFR (ischemic threshold $\leq 0.8$ ) in the assessment of LAD ischemia $(k=0.29$; fair concordance). At 4 months after revascularization, there was $77 \%$ agreement $(k=0.52$; moderate concordance) between the index CMR assessment of LAD ischemia and the follow-up LAD FFR. Concordance was improved at a LAD FFR ischemic threshold of $\leq 0.75$.

Conclusions: In this hypothesis generating study, baseline CMR assessment of LAD ischemia correlated better with the 4 months LAD FFR data (threshold $\leq 0.8$ ) as compared to the FFR measurements taken prior to RCA CTO revascularization. (Cardiol J 2022; 29, 1: 80-87)
\end{abstract}

Key words: chronic total occlusion, stress perfusion cardiovascular magnetic resonance, percutaneous coronary intervention

Address for correspondence: Dr. Richard E. Jones; BSc, MBChB, MRCP, Department of Cardiology, The Essex Cardiothoracic Centre, Basildon and Thurrock, University Hospitals NHS Foundation Trust, Basildon, SS16 5NL, United Kingdom, tel: +441268524900 ext. 4170, fax: +441268394127, e-mail: richard.jones34@nhs.net

Received: 28.10.2018 Accepted: 25.12.2019 Early publication date: 21.01.2020

This article is available in open access under Creative Common Attribution-Non-Commercial-No Derivatives 4.0 International (CC BY-NC-ND 4.0) license, allowing to download articles and share them with others as long as they credit the authors and the publisher, but without permission to change them in any way or use them commercially. 


\section{Introduction}

Stress perfusion cardiovascular magnetic resonance (CMR) and fractional flow reserve (FFR) are two frequently performed investigations in patients with suspected myocardial ischemia. FFR is the current gold standard for invasive functional assessment of coronary artery disease of intermediate severity and numerous randomized control trials have highlighted its prognostic value [1-3]. Furthermore, research has shown that perfusion CMR can accurately diagnose flow-limiting lesions as established by FFR [4-6].

Multiple studies have revealed that FFR measured in collateral donor vessels, in the presence of a chronic total occlusion (CTO), will increase after percutaneous revascularisation of the CTO [7-9]. This has important clinical implications; in the recent IMPACT-CTO study an increase in the predominant donor vessel FFR at 4 months following right coronary artery (RCA) CTO percutaneous coronary intervention (PCI) led to a functional reclassification in $18 \%$ of lesions at follow-up [7]. Thus, the ability of FFR to identify ischemia in a remote artery territory prior to revascularization of a CTO may not be robust.

Stress perfusion CMR has potential advantages in patients with multivessel disease in the presence of a CTO. CMR documents ischemia at the segmental myocardial level, in contrast to FFR which determines ischemia in a vascular territory. In the presence of a CTO, FFR in a donor artery is influenced not only by the degree of stenosis within the vessel, but also by the additional territory supplied by donor vessel collaterals to the CTO region. The resultant increase in flow and size of myocardial bed leads to a decrease in FFR and potentially a false positive result [8]. This raises the possibility that CMR may be a more accurate method of identifying ischemia in remote territories, which in turn could improve decision making and outcomes in patients with multivessel disease in the presence of a CTO.

In the present study an analysis was performed of patients enrolled in the IMPACT-CTO study to investigate the correlation between baseline stress perfusion CMR and serial FFR measurements of left anterior descending artery (LAD) remote territory ischemia, taken at baseline in the presence of an RCA CTO, and at 4 months after CTO PCI.

\section{Methods}

The full methodology and results for the IMPACT-CTO study (NCT02643940 clinical trials. gov number) had been reported previously [7]. In brief, 40 consecutive patients with an RCA CTO scheduled for PCI were recruited between October 2015 and November 2016. Inclusion criteria included symptomatic stable angina, RCA total occlusion of $\geq 3$ months duration, evidence of myocardial viability \pm ischemia on non-invasive testing, and visible collateral supply from a contralateral donor vessel. Myocardial viability on CMR was defined as $<50 \%$ of left ventricular wall hyperenhancement during delayed gadolinium imaging. Exclusion criteria included prior coronary artery bypass grafting, > 1 CTO vessel, and left main stem disease. The study was approved by the regional ethics committee (15/EE/0269) and all patients gave written informed consent. CTO PCI was performed with conventional techniques by experienced operators. FFR assessment of remote vessels was performed using a pressure wire (Philips Volcano Corporation, San Diego, California) before, immediately after, and at 4 months following RCA CTO PCI. Hyperemia was attained with an intravenous adenosine infusion administered via a femoral vein at a dose of $140 \mathrm{mcg} / \mathrm{kg} / \mathrm{min}$. Intracoronary nitroglycerine was administered before the physiological measurements were acquired. The pressure wire was normalized at the end of the guide catheter and passed to the distal aspect of the remote vessel. Two FFR ischemic thresholds of $\leq 0.8$ and $\leq 0.75$ were used in this sub analysis.

\section{CMR protocol}

Baseline perfusion CMR examinations were undertaken on a subset of patients in the IMPACT-CTO study in a 1.5-T scanner (Siemens MAGNETOM Aera). Standard cine steady-state-free-processing images were collected in three long axis views and multiple short axis slices. Perfusion data was acquired after a 3-4 min adenosine infusion $(140 \mu \mathrm{g}-210 \mu \mathrm{g} / \mathrm{kg} / \mathrm{min})$ and subsequent gadolinium-based contrast agent injection $(0.1 \mathrm{mmol} /$ $/ \mathrm{kg}$ at $6 \mathrm{~mL} / \mathrm{s}$ ). Three slices (basal, mid ventricular, and apical) were then obtained during the first pass using a TurboFLASH T1 weighted gradient echo sequence. Rest perfusion images were acquired after a period of $10 \mathrm{~min}$ and a second bolus of gadolinium contrast agent $(0.1 \mathrm{mmol} / \mathrm{kg}$ at $6 \mathrm{~mL} / \mathrm{s})$ was administered for the delayed enhancement imaging (assessed at an additional $7 \mathrm{~min}$ interval).

\section{CMR analysis}

The stress CMR data was analyzed by two experienced readers (SG and JND) blinded to the left coronary anatomy and background history. The myocardium was divided using a 16 -segment model (American Heart Association 17-segment model minus the apical cap). For the main analysis, the 
LAD was allocated into segments $1,2,7,8,13$, and 14. The RCA was assigned segments $3,4,9,10$, and 15 (all patients were RCA dominant) and the left circumflex artery was assigned segments 5,6 , 11,12 , and 16 . Visual assessment of LAD ischemia was determined by consensus agreement by the imaging consultants. Inducible perfusion defects were defined as delayed entry of contrast (persisting for more than 5 heart beats) in the absence of a scar. The cine sequences and delayed enhancement images were assessed simultaneously in order to evaluate for regional wall motion abnormalities and infarcted myocardium. A Siemens Syngo.via workstation was used for all analysis.

\section{Reassignment of coronary territories}

To assess the potential impact of variable coronary anatomy, one experienced interventional cardiologist (JRD) reviewed all angiographic data included in the sub analysis. Each myocardial segment subsequently reassigned (from the 16-segment model) to the specific coronary artery subtending that territory. The reassignment of perfusion territories was blinded.

\section{Statistical analysis}

Continuous data is presented as means \pm standard deviation. Categorical data is expressed as percentages. Percentage agreement and the kappa statistic was used to assess concordance between the perfusion CMR and FFR results (a kappa statistic of +1 signifying perfect agreement and a kappa statistic of -1 signifying full disagreement). Statistical analysis was performed using GraphPad.

\section{Results}

Twenty-six patients had baseline perfusion CMR testing and follow up remote artery FFR measurements at 4 months. All of these individuals were included in the final analysis. Average age was $62 \pm 9.8$ years, $89 \%$ were male, $19 \%$ had diabetes mellitus, and the mean LAD stenosis by quantitative coronary angiography (QCA) was $41 \% \pm 11.1 \%$. There was no significant difference in the hemodynamic parameters (central venous pressure, mean arterial pressure, and heart rate) during FFR assessment at baseline and follow-up. Full demographic data is presented in Table 1.

\section{LAD FFR analysis}

Sixteen $(62 \%)$ patients had a positive LAD FFR $(\leq 0.8)$ at baseline, $16(62 \%)$ patients had positive LAD FFR $(\leq 0.8)$ immediately after RCA
Table 1. Baseline demographic and angiographic data.

\begin{tabular}{lc}
\hline & N (\%) or mean \pm SD \\
\hline Demographic $(\mathbf{n}=\mathbf{2 6})$ & $23(85 \%)$ \\
Male & $62 \pm 9.8$ \\
Age [years] & $17(65 \%)$ \\
Previous MI & $10(38.5 \%)$ \\
Previous PCl & $17(65.4 \%)$ \\
Hypertension & $20(76.9 \%)$ \\
Hypercholesterolemia & $5(19.2 \%)$ \\
Diabetes mellitus & $5(19.2 \%)$ \\
Current smoker & $37.15 \pm 52.48$ \\
Angina duration [months] & $3(12 \%) / 10(38 \%) /$ \\
Angina CCS class (1/2/3/4) & $/ 13(50 \%) / 0(0 \%)$ \\
Estimated CTO duration & $224.38 \pm 392.16$ \\
[weeks] & \\
Angiographic details & $26(100 \%)$ \\
RCA CTO & $40.91 \pm 11.07$ \\
LAD stenosis on QCA [\%]
\end{tabular}

$\mathrm{SD}$ - standard deviation; $\mathrm{MI}$ - myocardial infarction; $\mathrm{PCl}$ - percutaneous coronary intervention; CCS - Canadian Cardiovascular Society; CTO - chronic total occlusion; RCA - right coronary artery; LAD - left anterior descending artery; QCA — quantitative coronary angiography

CTO PCI, and $12(46 \%)$ patients had a positive LAD FFR $(\leq 0.8)$ at 4 -month follow-up.

\section{Perfusion CMR analysis}

Perfusion CMR demonstrated RCA territory ischemia in $25(96 \%)$ patients and LAD ischemia in $8(31 \%)$ patients.

\section{CMR and FFR concordance}

in the assessment of LAD ischemia

There was $62 \%$ agreement between baseline CMR and FFR (cut off $\leq 0.8$ ) in the assessment of LAD ischemia ( $\mathrm{k}=0.29$; fair concordance) prior to RCA CTO PCI. 4 months after revascularization, there was $77 \%$ agreement $(\mathrm{k}=0.52$; moderate concordance) between the baseline CMR and the follow-up FFR (cut off $\leq 0.8$ ) assessment of LAD ischemia. In the cases of FFR and CMR discordance was at 4 months; 1 patient was CMR positive and FFR negative for LAD ischemia and 5 individuals were FFR positive and CMR negative for LAD ischemia (Table $2 \mathrm{~A}$ ).

$5 / 26$ (19\%) of patients had a positive LAD FFR $(\leq 0.80)$ prior to revascularisation and a negative LAD FFR $(>0.8)$ at 4 months. Perfusion CMR was negative for LAD ischemia in all of these 
Table 2. A. Concordance between baseline perfusion cardiovascular magnetic resonance (CMR) and serial fractional flow reserve (FFR) measurements (at an ischemic threshold of $\leq 0.80$ ); B. Concordance between baseline perfusion CMR and serial FFR measurements (at an ischemic threshold of $\leq 0.75$ ). The results were not affected by reassignment of coronary territories following blinded angiographic review.

\begin{tabular}{|c|c|c|c|c|}
\hline \multirow{3}{*}{$\begin{array}{l}\text { A } \\
\text { CMR result }\end{array}$} & \multicolumn{4}{|c|}{ FFR results (threshold of $\leq 0.80$ ) } \\
\hline & \multicolumn{2}{|c|}{ Prior to RCA CTO PCI } & \multicolumn{2}{|c|}{4 months following RCA CTO PCI } \\
\hline & LAD FFR negative & LAD FFR positive & LAD FFR negative & LAD FFR positive \\
\hline Negative for LAD ischemia & 9 & 9 & 13 & 5 \\
\hline Positive for LAD ischemia & 1 & 7 & 1 & 7 \\
\hline \multirow[t]{2}{*}{ B } & \multicolumn{4}{|c|}{ FFR results (threshold of $\leq 0.75$ ) } \\
\hline & \multicolumn{2}{|c|}{ Prior to RCA CTO PCI } & \multicolumn{2}{|c|}{4 months following RCA CTO PCI } \\
\hline CMR result & LAD FFR negative & LAD FFR positive & LAD FFR negative & LAD FFR positive \\
\hline Negative for LAD ischemia & 15 & 3 & 15 & 3 \\
\hline Positive for $L A D$ ischemia & 1 & 7 & 2 & 6 \\
\hline
\end{tabular}

RCA — right coronary artery; CTO — chronic total occlusion; $\mathrm{PCl}$ - percutaneous coronary intervention; LAD — left anterior descending artery

studies. Examples of concordant and discordant assessments of myocardial ischemia are shown in Figures 1 and 2.

At a lower FFR ischemic threshold of $\leq 0.75$, there was $85 \%$ agreement between CMR and FFR in the assessment of LAD ischemia prior to RCA CTO PCI ( $\mathrm{k}=0.66$; good concordance). At 4 months following RCA CTO revascularization, there was $81 \%$ agreement $(\mathrm{k}=0.56$; moderate concordance) between the baseline CMR and follow up FFR measurement of LAD ischemia at that decreased cut off (Table 2B).

In 22 patients, the LAD was the predominant donor vessel to the RCA CTO territory. In these individuals, there was $59 \%$ agreement between CMR and FFR (cut off $\leq 0.8$ ) in the evaluation of $\mathrm{LAD}$ ischemia prior to RCA CTO PCI $(\mathrm{k}=0.26$; fair concordance). At 4 months following RCA revascularization, there was $72 \%$ agreement $(\mathrm{k}=0.46$; moderate concordance) between the baseline CMR and the follow up FFR (cut off $\leq 0.8$ ) assessment of LAD ischemia. At a lower FFR threshold of $\leq 0.75$, there was $82 \%$ agreement between FFR and $\mathrm{CMR}$ results $(\mathrm{k}=0.61$; good concordance) prior to RCA CTO PCI. At 4 months after revascularization, there was $77 \%$ agreement $(\mathrm{k}=0.50$; moderate concordance) between the baseline CMR and follow up FFR results at this reduced threshold.

\section{Reassignment of coronary territories \\ Concordance between CMR and FFR was not affected by the reassignment of coronary ter-}

ritories following blinded angiographic review (Table 2A, B).

\section{Collateral vessel regression}

Twenty-two (85\%) patients in the sub analysis had evidence of full regression of their collateral vessels at follow up. One of the four patients who had persisting collateral circulation had discordant results at four months (LAD FFR 0.62 and CMR negative for LAD ischemia).

\section{Discussion}

The major findings of this study are:

- There appears to be only fair concordance between perfusion CMR and FFR (ischemic threshold $\leq 0.8$ ) in the assessment of LAD ischemia prior to RCA CTO revascularization;

- Baseline perfusion CMR appears to correlate better with the FFR assessment (cut off $\leq 0.8$ ) of LAD ischemia at 4 months post RCA CTO $\mathrm{PCI}$ as compared to before revascularization. This result appears to be due to the ability of a negative baseline CMR to identify patients with negative LAD FFR measurements at 4-month follow-up;

- Concordance in the assessment of LAD ischemia between perfusion CMR and pre-CTO PCI FFR appears to be improved at a lower ischemic threshold (cut off $\leq 0.75$ ). 


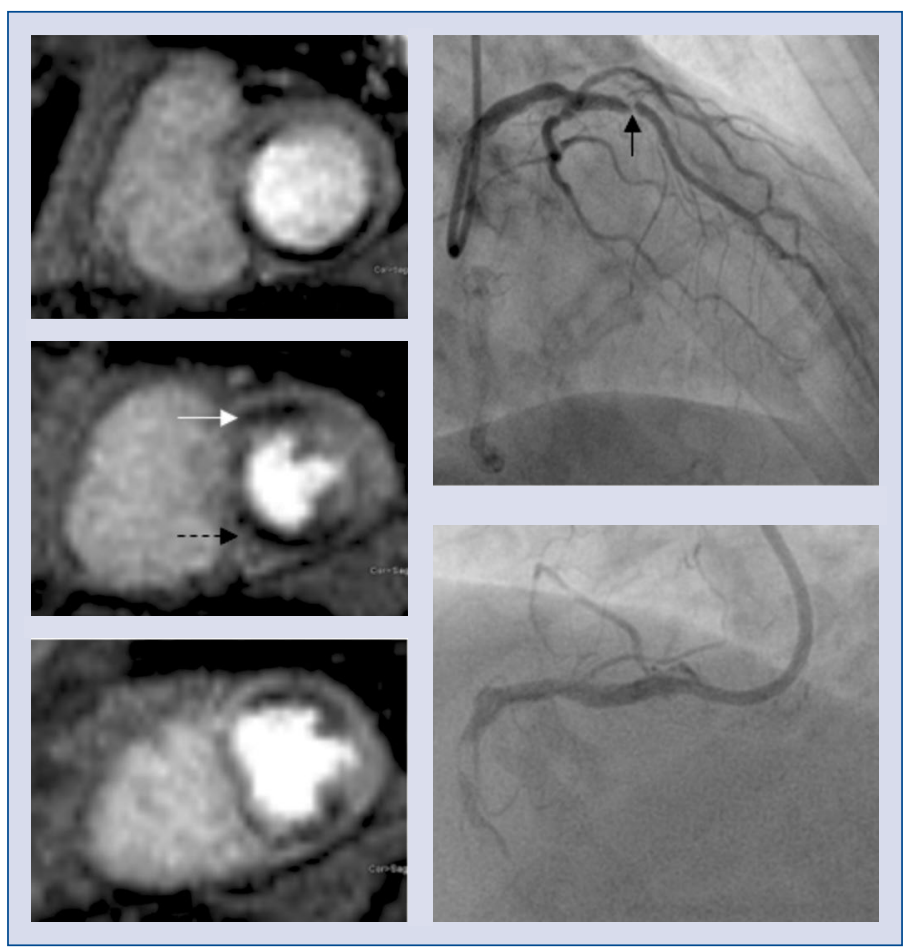

Figure 1. Concordant stress perfusion cardiovascular magnetic resonance (CMR) and fractional flow reserve (FFR) results. CMR imaging (left panel) demonstrating a perfusion defect in the left anterior descending (LAD) artery (e.g. white arrow) and right coronary artery (RCA; e.g. dashed arrow) territories. Coronary angiogram (right panel) demonstrating concordant findings with a severe LAD lesion (FFR 0.58 at baseline and 0.33 at 4 months; black arrow) and an occluded RCA.

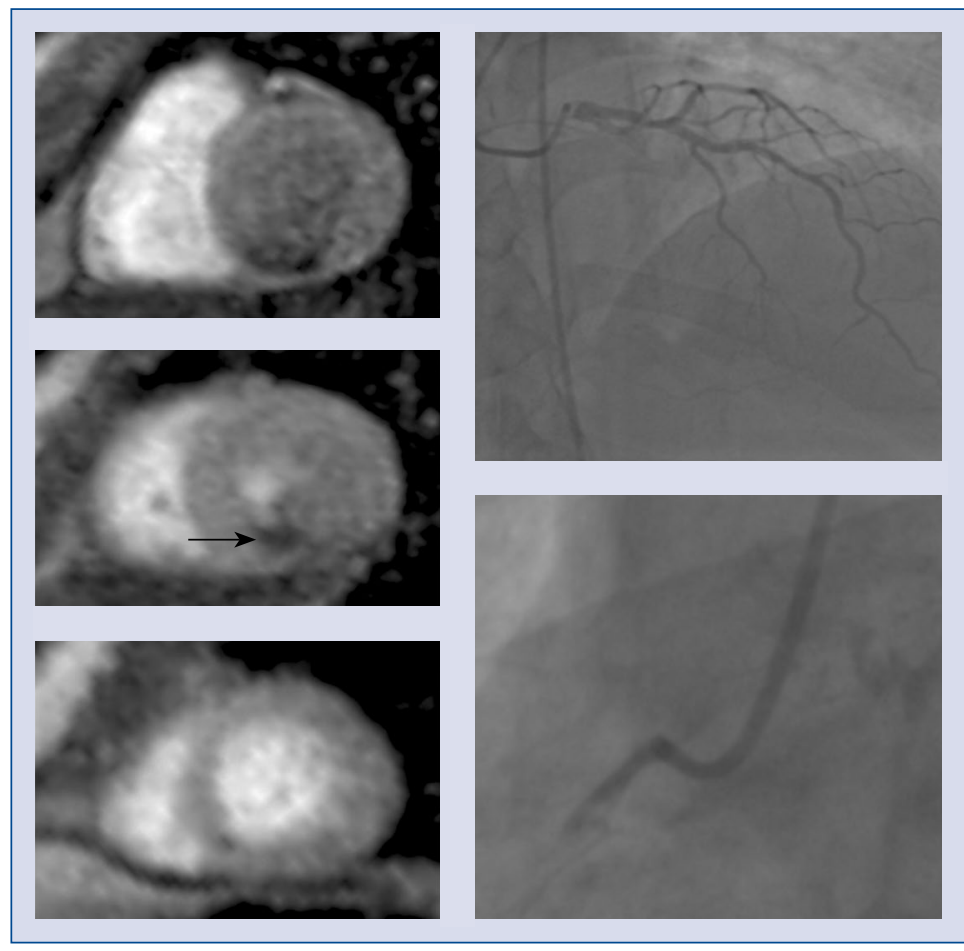

Figure 2. Discordant stress perfusion cardiovascular magnetic resonance (CMR) and baseline fractional flow reserve (FFR) result. CMR imaging (left panel) demonstrating a perfusion defect in the right coronary artery (RCA) territory (e.g. black arrow). Coronary angiogram (right panel) demonstrating discordant findings with an occluded RCA and a left anterior descending artery with an FFR of 0.78 at baseline and 0.82 at 4 months following RCA percutaneous intervention. 
Correlation between CMR and FFR in assessment of LAD ischemia in the context of an RCA CTO

In this sub analysis, there is only fair concordance between perfusion CMR and FFR assessment of LAD ischemia prior to RCA CTO PCI (using an FFR threshold of $\leq 0.80$ ). This result does not appear to be greatly affected by stratifying for the donor vessel or by the standard assignment of perfusion territories (i.e. the American Heart Association 17-segment model minus the apical cap). The majority of the discordance was due to patients with positive LAD FFR lesions and negative perfusion CMR scans (for LAD ischemia).

These results are similar to prospective studies assessing CMR and FFR in the assessment of multivessel disease (MVD). Hussain et al. [10] compared the diagnostic accuracy of perfusion CMR and FFR in patients with 2- and 3-vessel disease. They found fair concordance (at a per vessel basis analysis) between the two modalities and demonstrated that CMR either underestimated, or FFR overestimated the number of ischaemic territories in $33 \%$ of cases [10]. A further study by Nakamori et al. [11] again assessed the concordance of myocardial perfusion CMR to FFR in patients with both single, and MVD. In their study, visual assessment of ischemia by CMR (at a per vessel level analysis) had a sensitivity of $64 \%$ and a specificity of $79 \%$ against FFR in 2- or 3-vessel disease [11]. In contrast, in patients with single vessel disease, sensitivity of CMR was $83 \%$ and specificity was $95 \%$ (as compared to FFR) [11].

The etiology of the discordance found in the present sub analysis is likely multifactorial but will, in part, be due to inherent limitations of both modalities in individuals with a CTO. FFR influenced by myocardial mass subtended by the vessel being interrogated [12]. In patients with a collateralized CTO, coronary arteries not only supply their own territory, but also the myocardium of the occluded vessel. As such, the use of fractional flow to evaluate remote artery ischemia in such patients could have limitations and may lead to false positive results.

Stress perfusion CMR imaging could also have limitations in patients with MVD. Firstly, visual assessment of ischemia may be liable the same errors encountered with myocardial perfusion imaging (with single-photon emission computed tomography) in MVD. Myocardial perfusion imaging (MPI) relies on relative flow heterogeneity and as such, can encounter problems with 2 - or 3-vessel disease [13]. In these cases, MPI identifies the ter- ritories subtended by the most severe stenoses and has the potential to disregard perfusion defects in other myocardial segments [13]. Conversely, CMR has superior spatial resolution (i.e. can distinguish between subendocardial and transmural perfusion deficits) and therefore false negative results may potentially be avoided in MVD [11]. Indeed, sub analysis in the CE-MARC study revealed that triplevessel disease was not significantly associated with false negative perfusion CMR results [14].

In the case where FFR was negative and CMR was positive for LAD ischemia, there was no significant epicardial stenosis on quantitative coronary angiography. The CMR result may therefore have reflected microvascular ischemia and indeed, it is known that microvascular disease can alter the ability of FFR to assess the significance of epicardial disease [15].

\section{Concordance of baseline CMR and serial} FFR measurements in patients undergoing RCA CTO PCI; ischemic FFR threshold $\leq \mathbf{0 . 8}$

In this small hypothesis generating study, baseline perfusion CMR appears to correlate better with FFR in the assessment of LAD ischemia at 4 months post RCA CTO PCI as compared to before revascularization. This result appears to be driven by the ability of a negative baseline CMR to identify patients with negative LAD FFR measurements at 4-month follow-up.

These are interesting findings as the IMPACT-CTO study concluded that interpretation of baseline FFR measurements in vessels providing major collaterals to a CTO should be made cautiously [7]. In particular, when their FFR values were close to the ischemic cut off, the indices only became reliable after collateral vessel regression (i.e. at 4 months post PCI in the IMPACT-CTO study) [7]. In clinical practice however, revascularization decisions are often guided by index invasive coronary physiology of contralateral arteries in CTO patients. A 'gold standard' test would correctly identify significant epicardial stenoses independent of the altered coronary physiology of a collateralized CTO. The current sub analysis suggests that baseline CMR improved correlation with invasive data acquired at 4 months and as such, could act as a reliable tool in the evaluation of remote artery lesions prior to CTO PCI.

A recent study by Bucciarelli-Ducci et al. [16] assessed the use of CMR in the management of patients with a coronary CTO. As part of their study, remote territory myocardial perfusion reserve was measured (MPR) before and after CTO PCI (66\% 
of their study patients had MVD) [16]. At 3-month follow-up, there was no significant change in the remote territory MPR as compared to the assessment prior to CTO revascularization [16]. Another study by Cheng et al. [17] evaluated resting and hyperemic myocardial blood flow (MBF) by CMR in individuals undergoing CTO recanalization. They found that the remote segment MBF (resting or hyperemic) did not significantly rise at 6 months following CTO PCI as compared with baseline [17]. As with the present results, these studies suggest that CMR may be able to reliably assess the significance of bystander disease in patients with a CTO even prior to CTO revascularization.

It is unlikely that the failure of collateral regression significantly impacted the current results. Ongoing presence of donor vessels arising from the LAD could have affected the LAD FFR. In the present study however, only 4 patients had evidence of ongoing collateral circulation at 4 months. One of these patients had discordant results and this individual had a strongly positive LAD FFR (FFR 0.62) and a negative perfusion CMR for LAD ischemia. As such, it was felt that this was a false negative CMR result and not the consequence of residual LAD supply to the RCA territory.

\section{Concordance of baseline CMR and serial} FFR measurements in patients undergoing RCA CTO PCI; ischemic FFR threshold $\leq \mathbf{0 . 7 5}$

At a lower FFR ischemic threshold of $\leq 0.75$, the correlation between the two assessments of LAD ischemia improved. This is in keeping with a prior study which highlighted improved concordance when a reduced ischaemic cut-off for invasive physiological measurements was utilised in MVD [10]. The present results suggest that prior to CTO revascularization, a lower FFR ischemic threshold may be more appropriate in guiding the decision to revascularize the donor vessel.

\section{Limitations of the study}

This study has a number of limitations including its small number of subjects. Another main limitation is its design. Although CMR readers were blinded to the left coronary anatomy, they were aware that each patient had an RCA CTO. As such, there was a potential bias towards assessing for inferior ischemia. In addition, undertaking a repeat CMR at 4 months (to mirror the follow-up invasive measurements) would have allowed for a much improved analysis on the use of perfusion CMR in this cohort. Indeed, there are major limitations in comparing baseline CMR scans to FFR data collected 4 months following the index non-invasive test and a PCI procedure. As mentioned above however, a number of studies have performed serial perfusion CMRs in CTO patients $[16,17]$. Interestingly, these studies did not find evidence of significant variation in remote territory ischemia following CTO PCI $[16,17]$. Therefore, it may be fair to assume that the perfusion CMR appearance in the LAD territory would not have changed on follow-up imaging in the IMPACT-CTO cohort, in the absence of further revascularization. As such, baseline perfusion CMR examinations herein, may be a reliable comparator against the serial FFR measurements.

A further potential limitation is that qualitative assessment of CMR ischemia was performed in the current analysis. This does have the disadvantage of requiring a normal reference area of myocardium for visual comparison. Utilising a semiquantitative, or quantitative, approach to CMR analysis would have potentially provided a more robust assessment of inducible perfusion deficits. Finally, 2-dimensional perfusion CMR (as conducted in the present study) is limited by a lack of total myocardial coverage (e.g. conventionally three non-contiguous short axis myocardial slices, excluding the apical cap) [18]. Three-dimensional perfusion CMR is an exciting area of development which allows for whole-heart coverage and has been shown to have good diagnostic accuracy as compared to FFR [19].

Further studies incorporating all of the above methodological changes would enhance the strength of the ensuing results.

\section{Conclusions}

The IMPACT-CTO CMR analysis highlights the potential use of CMR in the work up of patients with borderline LAD lesions and an RCA CTO. Larger prospective studies are needed to assess whether baseline perfusion CMR is able to reliably act as a gatekeeper for revascularization in patients with LAD disease and an RCA CTO.

\section{Conflict of interest: None declared}

\section{References}

1. Zimmermann FM, Ferrara A, Johnson NP, et al. Deferral vs. performance of percutaneous coronary intervention of functionally non-significant coronary stenosis: 15-year follow-up of the DEFER trial. Eur Heart J. 2015; 36(45): 3182-3188, doi: 10.1093/ eurheartj/ehv452, indexed in Pubmed: 26400825. 
2. Tonino PAL, De Bruyne B, Pijls NHJ, et al. Fractional flow reserve versus angiography for guiding percutaneous coronary intervention. N Engl J Med. 2009; 360(3): 213-224, doi: 10.1056/ NEJMoa0807611, indexed in Pubmed: 19144937.

3. De Bruyne B, Pijls NHJ, Kalesan B, et al. Fractional flow reserve-guided $\mathrm{PCI}$ versus medical therapy in stable coronary disease. N Engl J Med. 2012; 367(11): 991-1001, doi: 10.1056/ NEJMoa1205361, indexed in Pubmed: 22924638.

4. Watkins S, McGeoch R, Lyne J, et al. Validation of magnetic resonance myocardial perfusion imaging with fractional flow reserve for the detection of significant coronary heart disease. Circulation. 2009; 120(22): 2207-2213, doi: 10.1161/CIRCULATIONAHA.109.872358, indexed in Pubmed: 19917885.

5. Lockie T, Ishida M, Perera D, et al. High-resolution magnetic resonance myocardial perfusion imaging at 3.0-Tesla to detect hemodynamically significant coronary stenoses as determined by fractional flow reserve. J Am Coll Cardiol. 2011; 57(1): 70-75, doi: 10.1016/j.jacc.2010.09.019, indexed in Pubmed: 21185504.

6. Bettencourt N, Chiribiri A, Schuster A, et al. Cardiac magnetic resonance myocardial perfusion imaging for detection of functionally significant obstructive coronary artery disease: a prospective study. Int J Cardiol. 2013; 168(2): 765-773, doi: 10.1016/j.ijcard.2012.09.231, indexed in Pubmed: 23102601.

7. Mohdnazri SR, Karamasis GV, Al-Janabi F, et al. The impact of coronary chronic total occlusion percutaneous coronary intervention upon donor vessel fractional flow reserve and instantaneous wave-free ratio: Implications for physiology-guided PCI in patients with CTO. Catheter Cardiovasc Interv. 2018; 92(3): E139-E148, doi: 10.1002/ccd.27587, indexed in Pubmed: 29569332.

8. Ladwiniec A, Cunnington MS, Rossington J, et al. Collateral donor artery physiology and the influence of a chronic total occlusion on fractional flow reserve. Circ Cardiovasc Interv. 2015; 8(4), doi: 10.1161/CIRCINTERVENTIONS.114.002219, indexed in Pubmed: 25805570.

9. Sachdeva R, Agrawal M, Flynn SE, et al. Reversal of ischemia of donor artery myocardium after recanalization of a chronic total occlusion. Catheter Cardiovasc Interv. 2013; 82(4): E453-E458, doi: 10.1002/ccd.25031, indexed in Pubmed: 23703834.

10. Hussain ST, Chiribiri A, Morton G, et al. Perfusion cardiovascular magnetic resonance and fractional flow reserve in patients with angiographic multi-vessel coronary artery disease. J Cardiovasc Magn Reson. 2016; 18(1): 44, doi: 10.1186/s12968-0160263-0, indexed in Pubmed: 27430288.

11. Nakamori S, Sakuma H, Dohi K, et al. Combined assessment of stress myocardial perfusion cardiovascular magnetic resonance and flow measurement in the coronary sinus improves prediction of functionally significant coronary stenosis determined by fractional flow reserve in multivessel disease. J Am Heart Assoc. 2018; 7(3), doi: 10.1161/JAHA.117.007736, indexed in Pubmed: 29432130.

12. Leone AM, De Caterina AR, Basile E, et al. Influence of the amount of myocardium subtended by a stenosis on fractional flow reserve. Circ Cardiovasc Interv. 2013; 6(1): 29-36, doi: 10.1161/CIRCINTERVENTIONS.112.971101, indexed in Pubmed: 23322740.

13. Melikian N, De Bondt P, Tonino P, et al. Fractional flow reserve and myocardial perfusion imaging in patients with angiographic multivessel coronary artery disease. JACC Cardiovasc Interv. 2010; 3(3): 307-314, doi: 10.1016/j.jcin.2009.12.010, indexed in Pubmed: 20298990.

14. Kidambi A, Sourbron S, Maredia N, et al. Factors associated with false-negative cardiovascular magnetic resonance perfusion studies: A Clinical evaluation of magnetic resonance imaging in coronary artery disease (CE-MARC) substudy. J Magn Reson Imaging. 2016; 43(3): 566-573, doi: 10.1002/jmri.25032, indexed in Pubmed: 26285057.

15. van de Hoef TP, Nolte F, EchavarrÍa-Pinto M, et al. Impact of hyperaemic microvascular resistance on fractional flow reserve measurements in patients with stable coronary artery disease: insights from combined stenosis and microvascular resistance assessment. Heart. 2014; 100(12): 951-959, doi: 10.1136/ heartjnl-2013-305124, indexed in Pubmed: 24727867.

16. Bucciarelli-Ducci C, Auger D, Di Mario C, et al. CMR guidance for recanalization of coronary chronic total occlusion. JACC Cardiovasc Imaging. 2016; 9(5): 547-556, doi: 10.1016/j. jcmg.2015.10.025, indexed in Pubmed: 27085432.

17. Cheng ASH, Selvanayagam JB, Jerosch-Herold M, et al. Percutaneous treatment of chronic total coronary occlusions improves regional hyperemic myocardial blood flow and contractility: insights from quantitative cardiovascular magnetic resonance imaging. JACC Cardiovasc Interv. 2008; 1(1): 44-53, doi: 10.1016/j. jcin.2007.11.003, indexed in Pubmed: 19393143.

18. Motwani M, Kidambi A, Sourbron S, et al. Quantitative three-dimensional cardiovascular magnetic resonance myocardial perfusion imaging in systole and diastole. J Cardiovasc Magn Reson. 2014; 16: 19, doi: 10.1186/1532-429X-16-19, indexed in Pubmed: 24565078.

19. Manka R, Wissmann L, Gebker R, et al. Multicenter Evaluation of Dynamic Three-Dimensional Magnetic Resonance Myocardial Perfusion Imaging for the Detection of Coronary Artery Disease Defined by Fractional Flow Reserve. Circ Cardiovasc Imaging. 2015; 8(5), doi: 10.1161/circimaging.114.003061. 